\title{
Interference Mitigation in WBANS: Challenges and Existing solutions
}

\author{
Samaneh Movassaghi*, Mehran Abolhasan*, and David Smith ${ }^{\dagger}$ \\ * School of Communication and Computing, Centre for Real-Time Information Networks (CRIN), \\ University of Technology, Sydney, NSW, Australia \\ $\dagger^{\dagger}$ National ICT Australia (NICTA) ${ }^{\ddagger}$, Canberra, ACT, Australia \\ Email: (seyedehsamaneh.movassaghigilani@student.uts.edu.au), (mehran.abolhasan@uts.edu.au), \\ and (david.smith@nicta.com.au)
}

\begin{abstract}
Wireless Body Area Networks (WBANs) are an exciting new networking technology developed in the recent years with advancements in wireless communication, integrated circuits and Micro-Electro- Mechanical Systems (MEMs). They consist of a number of sensor nodes that are placed in or around the human body. However, their practical deployment requires addressing numerous challenges. WBANs face many stringent requirements in power, bandwidth, and network lifetime which need to be taken into serious consideration in the design of different protocols. In this paper, we investigate the importance of interference mitigation amongst coexisting Wireless Body Area Networks (WBANs). Since, a WBAN is most likely to encounter other WBANs, inter-WBAN interference and scheduling is of utmost importance.
\end{abstract}

Index Terms-Wireless Body Area Networks, IEEE 802.15.6, Interference Mitigation, Interference Avoidance

\section{INTRODUCTION}

Co-channel interference mitigation in WBANs is quite challenging due to the large density, high mobility and the uncoordinated nature of WBANs. Interference can be larger than the signal even in cases where two WBANs are far from each other. This is logical because of the large variation of channel gain with body movement which implies the link strength is independent of the distance. The mobility of WBANs makes interference even more challenging as WBANs can move into each others range and result in a large density of people with their WBANs in each others coexistence [1]. Coexistence has shown to provide much severe effects for higher data rates. Additionally, in most application WBANs are rational and self-interested which means they do not cooperate with each other to make power decisions that implies each WBAN independently chooses its transmission power based on its belief of other WBANs' choice [2].

The most valuable resource of a WBAN is energy which can be easily wasted by inter-network interference which reduces the SINR value and leads to throughput degradation. In order to maintain the minimum acceptable link quality, the transmit power should be limited to minimize the interference level and save battery life [3]. Thus, interference mitigation schemes aim to decrease the average transmit power using link adaptation

\footnotetext{
$\ddagger$ NICTA is funded by the Australian Government as represented by the Department of Broadband, Communications and the Digital Economy and the Australian Research Council through the ICT Centre of Excellence program
}

mechanisms whilst maintaining link quality at the cost of lower data rate or throughput [3]. A total of $70 \%$ of the total power consumption of off-the-shelf sensor nodes is related to the transmission power of their wireless transceivers. This can be even more challenging in the case of WBANs as battery replacement or recharging can be quite uncomfortable [4]. The scarce constraint of energy in WBANs requires simple, robust, intelligent and light solutions for these networks to cope with the small processing power and small memory of their devices.

The IEEE 802.15.6 working group has defined new PHY and MAC layer proposals for WBANs that provides ultralow power, low cost, low complexity and short range wireless communication in or around the human body. The proposed standard requires the system to function properly within the transmission range of up to 3 meters when up to 10 WBANs are co-located [5]. It also has to support 60 sensors in a $6 \mathrm{~m}^{3}$ space (256 sensors in a $3 m^{3}$ cube ) [5]. Thus, there is a large possibility of interference amongst WBANs in each others coexistence. The interference link is most likely to be from a device on some other person (off-body) and the signal link is most likely between two devices on one person (on-body) [1]. In cases where the main source of channel dynamics is subject to movement since the two objects are not synchronised, signal and interference links are uncorrelated and statistically independent when considered over a large period of time in the order of 100's. Whilst, in cases where mobility is not the main reason for channel dynamics (over a short period of time), signal and interference links are correlated and display similar characteristics [6].

Unfortunately, interference mitigation schemes proposed for other networks cannot be deployed in WBANs because of the following differences. A WBAN mainly has more frequent topology changes and a higher moving speed whilst a WSN has static or low mobility scenarios. WBANs are similar to MANETs in terms of the moving topology with group-based movement rather than node-based movement. Thus, due to the group-based movement and high mobility nature of WBANs they are not similar to WSNs or MANETs which implies their interference mitigation approaches cannot be used for WBANs [7]. In addition, due to the limitations of WBANs in terms of cost, size and energy consumption, the function of each sensor node needs to be very simple. Thus, advanced 
antenna techniques cannot be used for interference mitigation in WBANs [8]. Additionally, interference mitigation proposals for cellular networks cannot be deployed in WBANs due the following reasons. For one thing, power control is not convenient for WBANs due to their low energy consumption and simple structure whilst it is a feasible method in cellular networks. Additionally, the location of a mobile station is usually uniformly distributed in cellular networks whilst nodes in WBANs are deployed more densely. Also, neighbor networks in cellular networks adjoin with each other due to the network coverage requirement, whilst a gap between WBAN networks is acceptable to mitigate the mutual interference [8].

The rest of the paper is organized as follows. Section II describes Intra-WBAN and Inter-WBAN Interference. The interference mitigation schemes proposed thus far for WBANs are presented in Section III. Section IV concludes the paper.

\section{INTRA-WBAN AND INTER-WBAN INTERFERENCE}

Based on the proposed IEEE 802.15.6 standard, nodes in a single WBAN can avoid interference by using multiple access techniques such as time division [3]. Thus,interference is not an issue for intra-WBAN communication. Generally interference occurs when no-coordination exists amongst multiple WBANs that coexist in each other's vicinity [3]. In cases where co-located WBANs use the same channel (similar frequencies), transmissions can conflict; as the active periods can overlap [9]. Thus, WBANs operating at the same frequency should be synchronized. In [1], the performance of three multiple access schemes, namely CDMA, FDMA and TDMA for inter-network interference has been investigated using real-world interference measurements in terms of Bit Error Rate (BER), Statistics of signal to interference and noise ratio (SINR) and probability of collision. TDMA and FDMA have shown to be more efficient for interference mitigation whilst in the case of CDMA, WBANs have a high chance of collision as no set of codes can maintain orthogonality and may transmit over the same time and frequency in entirely asynchronous systems. TDMA has $N_{c}-1\left(N_{c}\right.$ refers to the number of orthogonal frequency channels ) times shorter transmission time than FDMA for the same number of bits. Since, power consumption is important in WBANs, the lower the operating time the lower the overall power consumption. Most contention-based protocols that use CSMA/CA utilize Clear Channel Assessment (CCA) to specify the status of the channel. However, the high path loss inside and outside WBANs does not guarantee this approach [10]. Scheduled based approaches like TDMA are efficient for CCA problems and traffic correlation. However, as all sensors in TDMA approaches must receive periodic control packets to synchronize their clocks extra energy is consumed for their periodic synchronization [10].

Moreover, with the increase in the number of WBANs that can coexist in short proximity of each other, the communication link can suffer performance degradation. Even in cases where small number of WBANs are deployed in each other's vicinity, the received signal strength of the interfering signal can be quite high which affects the performance of a particular WBAN [3]. In fact, WBANs can practically either exchange information such as channel gain, interference and current transmission power or collect this information through their own measurements [2].

The sources of interference may be from co-existing WBAN networks or non-WBAN networks. A reasonable assumption is to consider the overall interference that appears at the intended receiver node as white which means the intended WBAN network being observed acts as a lognormal fading channel with additive white Gaussian noise. According to the standard, in cases where interference occurs, the packet can be retransmitted during a certain time period before being considered lost. However, there is a tradeoff between the throughput requirement and energy consumption [9]

Nodes in WBANs have stringent energy constraints and require low power techniques which can be achieved by an appropriate choice of the MAC as it has a key role in defining the energy consumption. However, the proposed MAC protocols have mainly focused on enhancing throughput, latency and bandwidth utilization whilst not considering the major requirement of energy conservation [10]. The superframe structure of the WBAN MAC is shown in Figure. 1 and consists of the following four periods: control period, Contention Access Period (CAP), Contention Free Period (CFP) and inactive period. CAP initiates after GTS requests and topology management and is controlled by the CSMA-CA algorithm. CFP initiates at the end of the CAP and consists of a number of Guaranteed Time Slots (GTS) which can be assigned by the coordinator to the sensors based on TDMA [9, 11]. Since all WBANs are considered to use similar superframe structures and interWBAN superframe synchronization is achieved before transmission, a collision never occurs between one WBAN's data frame and another WBAN's control frame. Whilst, interference can occur between different WBAN's data frames in the CFP [9]. A packet in the superframe can be lost either via collision or buffer overflows. Collisions occur in the GTS slot where no carrier sensing is available. Buffer overflows occur in cases where CSMA-CA delays the transmissions because of the nonfree channel. Thus, CSMA/CA avoids collisions but fills the buffer whilst GTS does not avoid collision but does not fill the buffer [9].

Coexistence amongst WBANs mainly leads to beacon loss and data loss. Since beacon transmissions do not use carrier sensing, beacons of coexisting WBANs may collide with each other. In this case a beacon is lost; so the sensors lose synchronization and must not transmit in that superframe [12]. Depending on the mode of operation, data loss can occur when a number of WBANs coexist. For instance, life critical data require retransmission and acknowledgements. However, the lack of clear channel assessment and the inflexible nature of GTS approaches leads to inefficient consequences in the period of coexistence [12]. 


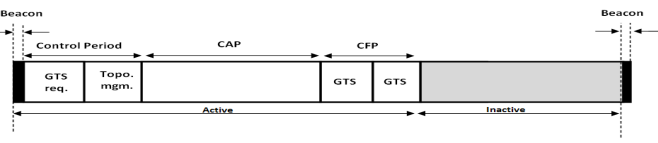

Fig. 1. WBAN MAC

\section{Proposed Interference Mitigation Approaches}

The proposed scheme for interference mitigation in WBANs can be divided into two categories: 1) interference reduction techniques, 2) interference avoidance techniques. In interference reduction schemes, different devices are possibly transmitting at the same time, but with different power, modulation scheme, data rate or phase. The aim of these approaches is mainly to minimize the interference level at the receiver by optimizing the system parameters such as power, data rate and some other physical layer parameters. In interference avoidance techniques, the coordinators of different WBANs attempt to assign orthogonal channels to each device in the network, thus avoiding the interference with the cost of lower system throughput.

\section{A. Interference Reduction Techniques}

Efficient power control mechanisms are used to maintain the link quality (signal to interference noise ratio or strength level). More specifically, the power quality is controlled to suffice the desired link quality. This scenario is even worse in cases where interference is quite significant; for instance in cases where other coexisting wireless networks have a high transmit power. For instance in the case where the signal cannot be clearly recovered by the intended receiver, this approach will increase the power level of the relevant transmitter to adjust the required link quality whilst causing interference for other networks in its vicinity. Therefore, nodes at approximate WBANs also increase their transmit power which degrades the link quality at the intended receiver and no links would be able to operate with an acceptable quality. However this approach is not convenient for use in WBANs due to postural body movements and the mobility of nearby WBANs [3]. In [4], a dynamic power control mechanism using reinforcement learning is proposed for interference mitigation in WBANs.

One other approach is to use advanced signal processing techniques to cancel interference. However, this approach cannot be practically used in WBANs. For one thing, these interference mitigation methods require knowledge of the channel between the receiver and each of the interferers; whilst such a accurate estimation of the channel condition is very challenging in WBANs. Additionally, this approach requires a receiver with high computational complexity which is not practical in the case of WBANs with dense deployment of nodes, specially as most WBAN nodes are battery-driven and require prolonged lifetime for one's own comfort.

In [3], adaptive modulation, adaptive data rate and adaptive duty cycle are proposed towards interference mitigation in wireless body area networks. These method have low complexity which is convenient for use in WBANs due to their small size and low computational capability. An Interference Mitigation Factor (IMF) is also introduced to evaluate the performance of the proposed schemes which is defined as the reduction of the transmit power level obtained by using an interference mitigation method compared to the original operation mode. It is a function of channel condition and SINR [3]. However, implementation complexity has not been considered and the effectiveness of these schemes for multiple WBANs has not been investigated. For adaptive modulation, a set of MPSK schemes such as BPSK, QPSK and 8PSK can be used as they have the same detection mechanism at the receiver. With a pre-defined BER, the SINR can be obtained from the channel conditions. 8PSK is used to gain higher bit rate at higher SINR values, whilst QPSK can be used when the transmit power is between a certain threshold. BPSK is used in cases where SINR is lower than the lower threshold [3]. For adaptive data rate, the date rate is divided into a number of steps between its maximum $\left(R_{\max }\right)$ and minimum value $\left(R_{\text {min }}\right)$. In the normal mode, the date rate operates at it maximum value and changes by comparing its target SINR and weighted sum of SINR values. The weighted sum may reduce the variations in the SINR. In terms of adaptive duty cycle, the duty cycle is divided into a number of steps between its maximum and minimum values. Normally, the duty cycle is set at its maximum value and changes by comparing its target SINR and its weighted sum of SINR.

In [2], a non-cooperative power control game is proposed for inter-network interference mitigation in WBANs. The proposed method aims to maximize the overall system throughput using the minimum power and applies a power pricing mechanism to minimize the transmission power of each user and maximizes the total transmission rate. An adaptive pricing mechanism is also proposed to dynamically adapt to changes in power budgets and channel gains; where the higher battery charge the lower the price associated to it. This way users with low power budgets and bad channel conditions are penalized whilst users with good channel conditions and high power budgets take advantage of their good conditions. As there are issues with accessing information regarding channel gain, their heavy cost and their overload on the system to measure it as well as exchanging it, the authors consider using variations in SINR which is less expensive and much suitable.

The authors in [13], proposed Interference Cancelation with Interrupted Transmission (ICIT) for a two-diversity branch receiver. ICIT uses the amplitude and phase of the interference known at one instance to combine the two received signals such that the interference signal at one branch is equal and out of phase at the other branch. In this approach, a predefined algorithm switches the desired signal off at regular intervals. Therefore, the amplitude and phase of the two interfering signals have to be calculated at the two receiving antennas. Thus, the desired signal transmitter is switched off at regular intervals which implies that at a certain distance only the phase and amplitude of the interference signal is measured. This value is then used to calculate the a weight vector for the next time interval until the required signal is interrupted. 


\section{B. Interference Avoidance Techniques}

Channel switching can be used as an approach towards interference mitigation in WBANs in cases where the amount of non-overlapping channels is higher than the number of coexisting WBANs so that a unique channel is assigned to each WBAN [9]. IEEE 802.15.4 has 16 non-overlapping channels at $2.4 \mathrm{GHz}$. Thus, switching is a convenient approach in this frequency and 16 WBANs can coexist with each other at this frequency. However, Bluetooth or IEEE 802.11 (WiFi) reduce the available interference-free channels.

In [7], a random incomplete coloring (RIC) with high spatial reuse and low complexity has been proposed for interference mitigation in WBANs, where interference avoidance is modeled as a graph coloring problem. This approach allows for higher spatial reuse than when a minimum of $k$ colors are used by allowing partial vertices to be left uncolored. The uncolored nodes represent nodes with no transmissions which implies no interference. Therefore, a subgraph of the main graph can be colored excluding the uncolored vertices. For data transmission, first the coordinator of the WBAN negotiates with other WBANs that are in its interfering range and assigns reserved resources to them accordingly. The end nodes of the WBAN only wake up when receiving beacon messages that carry the preregularized transmission schedule of the coordinator or when transmitting vital signals following the schedule towards the coordinator.

In [10], a traffic adaptive MAC protocol,namely (TaMAC) is proposed for WBANs which considers the traffic information of nodes in WBANs and the coordinator adjusts the duty cycle of the nodes based on their traffic pattern. Therefore, nodes with low duty cycles need not receive frequent synchronization and control packets if they do not any data to send transmit or receive. TaMAC aims to provide real-time health monitoring, tolerable delay, scalability, low power consumption, collision free transmission with TDMA and desirable QoS for all types of traffic. It classifies the traffic into normal, emergency traffic and on-demand. TaMAC uses two channel access mechanisms based on the type of the traffic, where a wakeup radio mechanism is used to reliably accommodate on-demand and emergency events and a traffic-based wakeup mechanism is used for emergency and on-demand traffic. This suffices the low energy consumption requirements of WBANs as it uses a separate control channel together with the data channel. A traffic-based wakeup table is built that stores the ID and traffic pattern of all the nodes. Thus, the nodes remain asleep unless they need to send or receive data. When two nodes have the same traffic pattern, resources are allocated to the node with the higher priority. In cases where two nodes have the same traffic pattern and the same priority, resources are allocated to the node with minimum data volume. This protocol has shown to outperform SMAC, 802.15.4 MAC and WiseMAC protocols in terms of delay and power consumption.

\section{Brief Comparison}

In comparison, interference avoidance schemes can achieve a higher SINR level compared to interference reduction schemes, but their throughput is usually lower. Moreover, in terms of computational complexity, interference avoidance techniques requires less complex receivers but extra cooperation between coordinators is inevitable. In interference reduction schemes, the receiver has a more complex decoder because it needs to decode several messages with different levels of SINR, data rate and power; but since cooperation is not performed between coordinators lower transmission overhead can be achieved by these approaches.

\section{CONCLUSION}

In this paper we have presented the challenges of deploying multiple WBANs in each others vicinity and the interference mitigation schemes proposed thus far for WBANs. Some of the challenges of WBANs have been considered in these proposals however; their practical deployment requires further research and investigation. The future vision of WBANs is to allow for reliable, cost-effective and energy efficient communication amongst all co-located WBANs.

\section{REFERENCES}

[1] A. Zhang, D. B. Smith, D. Miniutti, L. W. Hanlen, D. Rodda, and B. Gilbert, "Performance of piconet co-existence schemes in wireless body area networks," in Wireless Communications and Networking Conference (WCNC), 2010 IEEE. IEEE, 2010, pp. 1-6.

[2] R. Kazemi, R. Vesilo, E. Dutkiewicz, and G. Fang, "Inter-network interference mitigation in wireless body area networks using power control games," in Communications and Information Technologies (ISCIT), 2010 International Symposium on. IEEE, 2010, pp. 81-86.

[3] W.-B. Yang and K. Sayrafian-Pour, "Interference mitigation for body area networks," in Personal Indoor and Mobile Radio Communications (PIMRC), 2011 IEEE 22nd International Symposium on. IEEE, 2011, pp. 2193-2197.

[4] R. Kazemi, R. Vesilo, E. Dutkiewicz, and R. Liu, "Dynamic power control in wireless body area networks using reinforcement learning with approximation," in Personal Indoor and Mobile Radio Communications (PIMRC), 2011 IEEE 22nd International Symposium on. IEEE, 2011, pp. 2203-2208.

[5] IEEE standard for local and metropolitan area networks part 15.6: Wireless body area networks. IEEE Std 802.15.6-2012.

[6] A. Zhang, L. W. Hanlen, D. Miniutti, D. Rodda, and B. Gilbert, "Interference in body area networks: Are signal-links and interference-links independent?" in Personal, Indoor and Mobile Radio Communications, 2009 IEEE 20th International Symposium on. IEEE, 2009, pp. 456460 .

[7] S. Cheng and C. Huang, "Coloring-based inter-wban scheduling for mobile wireless body area network," 2013.

[8] X. Wang and L. Cai, "Interference analysis of co-existing wireless body area networks," in Global Telecommunications Conference (GLOBECOM 2011). IEEE, 2011, pp. 1-5.

[9] P. R. Grassi, V. Rana, I. Beretta, and D. Sciuto, "B2 IRS: A technique to reduce BAN-BAN interferences in wireless sensor networks," in Wearable and Implantable Body Sensor Networks (BSN), 2012 Ninth International Conference on. IEEE, 2012, pp. 46-51.

[10] S. Ullah and K. S. Kwak, "An ultra low-power and traffic-adaptive medium access control protocol for wireless body area network," J. Med. Syst., vol. 36, no. 3, pp. 1021-1030, Jun. 2012. [Online]. Available: http://dx.doi.org/10.1007/s10916-010-9564-2

[11] L. Wang, C. Goursaud, N. Nikaein, L. Cottatellucci, and J. Gorce, "Cooperative scheduling for coexisting body area networks," 2012.

[12] M. Deylami and E. Jovanov, "Performance analysis of coexisting ieee 802.15. 4-based health monitoring wbans," in Engineering in Medicine and Biology Society (EMBC), 2012 Annual International Conference of the IEEE. IEEE, 2012, pp. 2464-2467.

[13] I. Khan, Y. I. Nechayev, K. Ghanem, and P. S. Hall, "BAN-BAN interference rejection with multiple antennas at the receiver," Antennas and Propagation, IEEE Transactions on, vol. 58, no. 3, pp. 927-934, 2010. 